Research Paper

\title{
Gastrointestinal Infection with Mexican TcI Trypanosoma cruzi strains: Difierent Degrees of Colonization and Diverse Immune Responses
}

\section{Bertha Espinoza ${ }^{\bowtie}$, Natalia Solorzano-Domínguez, Andrea Vizcaino-Castillo, Ignacio Martínez, Ana L. Elias-López and José Antonio Rodríguez-Martínez.}

Departamento de Inmunología. Instituto de Investigaciones Biomédicas. Universidad Nacional Autónoma de México. Mexico City 04510.

Corresponding author: Bertha Espinoza. Phone: 525562289 43. Email: besgu@biomedicas.unam.mx

( ) Ivyspring International Publisher. This is an open-access article distributed under the terms of the Creative Commons License (http://creativecommons.org/ licenses/by-nc-nd/3.0/). Reproduction is permitted for personal, noncommercial use, provided that the article is in whole, unmodified, and properly cited.

Received: 2011.09.01; Accepted: 2011.10.01; Published: 2011.11.01

\begin{abstract}
Mexican Ninoa and Queretaro (Qro) Tcl strains of Trypanosoma cruzi have shown different degrees of virulence, and the two strains produce heterogeneous immune responses in the hearts of infected mice. This work shows that the same strains can invade the intestine by an intraperitoneal route and establish an infection, mainly in the colon. The three segments of the small intestine (duodenum, jejunum and ileum) were infected to a lesser degree than the colon. Despite the fact that parasites were predominantly found in the colon, an obvious inflammatory reaction was observed in the submucosal layer along the entire intestinal tract, with the virulent Qro strain causing significantly more areas of higher immune infiltration. A clear recruitment of CD4+ and CD8+ T lymphocytes to the mesenteric ganglia was observed during infection with the virulent strain. Macrophages were also differentially distributed in the gastrointestinal tract. These later cells infiltrated fewer amastigote nests in the mice infected with the Qro strain than in the mice infected with the Ninoa strain. When IFN- $\gamma$, TNF- $\alpha$, and IL-4 levels were measured, an increase in these cytokines was observed compared with the uninfected mice. The role of these inflammatory reactions in the pathogenesis of Chagas enteropathy is also discussed in this paper.
\end{abstract}

Key words: Intestinal infection, Mexican Trypanosome cruzi strains

\section{INTRODUCTION}

Chagas' disease is caused by the protozoan parasite Trypanosoma cruzi, and it affects as many as 10 to 16 million people in North and South America, where it represents a major public health problem [1]. Recently, however, due to the emigration of people from endemic areas, cases of Chagas'disease have been reported in the USA, Canada, Australia, Japan and Europe, extending the range of this parasitic disease throughout the developed world $[2,3]$. T. cruzi is a parasite with high genetic diversity, and it has been grouped into six discrete typing units (DTUs), T. cruzi I to VI [4]. It is known that the TcI DTU is predomi- nant in Central America, the northern region of South America and Mexico [5, 6, 7, 8]. Mexican isolates from humans and from vector insects have been primarily found to be TcI, and these isolates displayed heterogeneous processes of metacyiclogenesis and infection in vitro [9]. TcI strains are likely to be the strains that cause the clinical manifestations observed in Mexico [10], where it has been shown that cardiac manifestations are the main symptoms presented by patients with Chagas' disease [11]. The course of infection with $T$. cruzi includes an acute phase, during which the parasite infects a wide variety of tissues, which 
can include the heart, spleen, nervous system and intestinal tract. Parasites are also found in the circulating blood. The acute phase then evolves into a chronic phase, in which most patients are asymptomatic, presenting the so-called indeterminate phase. About $30 \%$ of infected individuals will develop the chronic manifestations of the disease, such as cardiac arrhythmia and cardiomyopathy [12]. Chagasic enteropathy is one manifestation that may occur during the chronic phase of Chagas' disease, probably as a result of the destruction of the myenteric plexus, which is located in the muscular layer of the small intestine [13]. Intestinal infection presents as a variety of clinical symptoms, ranging from mild difficulty swallowing to severe distension of the intestinal structure [14].

This last pathological state can be responsible for dyspeptic syndrome, intestinal pseudo-obstruction or bacterial overgrowth; it can also cause altered motility, which involves hypertrophy and dilatation, and causes megaduodenum, megajejunum, megaileum, the classic megacolon or megaesophagus [15].

It is well known that the three portions of small intestine (duodenum, jejunum and ileum) and the large intestine, which are involved in Chagasic enteropathy, have different structures and functions, as well as differences in the immune response. This compartmentalization of the immune system allows for complex interactions between a given site within the intestine and the parasite antigens. The mucosa has different characteristics in the different sections of the gastrointestinal tract. The small intestine contains a greater number of mucosal mast cells, which are a component of the Th2 response. Another difference is that the proximal small intestine (duodenum and jejunum) contains intraepithelial lymphocytes, which have a greater proliferative capacity, and contains fewer lymphoid follicles than the distal small intestine (ileum). The ileum contains not only more lymphoid follicles but also a greater number of Peyer's patches, which are sites of $\mathrm{T}$ and $\mathrm{B}$ cell production that contain antigen presenting cells and are also a potent source of cytokine and chemokine secretion. These differences might cause the three sections of the small intestine to generate different immune responses to the parasite $[16,17]$.

It is possible that Mexican TcI strains are able to infect the intestinal tract; however, there have been few reports until now of the presence of Mexican strains in the gut, and in those few reports, the genetic types of the strains were not identified. It has been suggested that there is a chronic inflammation of the mesenteric ganglia of infected individuals who develop gastrointestinal symptoms, but no information on such a response has been reported for Mexican strains. The aim of this work was to evaluate the infection of the intestinal tract during an intraperitoneal infection and to investigate the immune response induced by Mexican T. cruzi TcI strains in the different segments of the intestine.

\section{MATERIALS AND METHODS}

\section{Parasites}

The trypomastigote forms of Mexican strains of T. cruzi were obtained from the blood of infected mice. The following two Mexican strains of the parasite were used: Ninoa (MHOM/MX/1994/Ninoa) and Queretaro (TBAR/MX/0000/Queretaro) (Qro), which both belong to the TcI group [5,9]. These strains have been shown to have different levels of virulence. Ninoa is a non-virulent strain, while the Qro strain kills $60-100 \%$ of infected mice. Both strains infect the heart of Balb/c mice and induce a systemic and cardiac differential immune response [18].

\section{Experimental Animals}

Female Balb/c mice, 6-8 weeks of age, were provided by the Animal House at the Instituto de Investigaciones Biomedicas of Universidad Nacional Autonoma de Mexico (UNAM, Mexico city, Mexico). Mice were maintained under pathogen-free conditions and were given food and water ad libitum. Groups of mice were intraperitoneally infected with $1 \times 10^{4}$ blood trypomastigotes (BT). Parasitemia in the blood was measured every third day in a Neubauer chamber using microscopic observation. All animal research was performed in accordance with the guidelines of the Instituto de Investigaciones Biomedicas ethics committee.

\section{Histopathological Experiments}

Twenty-four mice in two independent experiments were used for the histological analysis; groups of eight mice were infected intraperitoneally with $1 \mathrm{X} 10^{4} \mathrm{BT}$ of the Ninoa strain, Qro strain or with PBS as a control. Tissues were collected at 15, 21 and 90 days post infection (pi). These time points were chosen because, using this inoculum of parasites, at day $15 \mathrm{pi}$, an increase of number of parasites are detected in the blood, and can be detected in the heart, of infected mice. At 21 days pi, the maximum level of parasites is present in the blood, and at 90 days, the chronic phase of the infection begins, using this inoculum of parasites [18].

The following tissues were collected: duodenum, jejunum and ileum (from the small intestine), colon and heart. The tissues were fixed in $4 \%$ paraformal- 
dehyde and embedded in paraplast X-tra (SIGMA-Aldrich Co., St. Louis, MO, USA). Serial sections of $5 \mu \mathrm{m}$ thickness were prepared from non-consecutive areas, stained with hematoxylin and eosin (H-E) and examined using an optical microscope (Nikon, model 022893).

\section{Immunohistochemistry}

The presence of parasites in the tissue was determinate by immunohistochemistry. Sections from the intestine were deparaffinized and rehydrated through serial passes in alcohols. They were then incubated with $2 \%$ bovine serum albumin (BSA) (SIGMA-Aldrich) for $60 \mathrm{~min}$, and each section was subsequently incubated with $50 \mu \mathrm{L}$ (diluted 1:1,000) of rabbit serum specific to $T$. cruzi (previously produced in our laboratory), at $4^{\circ} \mathrm{C}$ overnight. Pre-immune serum was used as a negative control. Each section was washed three times with PBS/1\% BSA, followed by an incubation with fluorescein-conjugated goat anti-rabbit IgG diluted 1:100 (anti-IgG FITC; Sigma Immuno Chemicals, St Louis, MO, USA) for $60 \mathrm{~min}$ at room temperature. Each section was then visualized using a fluorescence microscope.

For macrophage localization, a 1:50 dilution of anti-F4/80 biotinylated antibody or the IgG2b isotype control (Caltag Laboratories, Buckingham, UK) were used. Streptavidin-phycoerythrin conjugate (Caltag Laboratories) diluted 1:500 was then added. DAPI (Molecular Probes, Eugene, Oregon, USA) was used to stain the nucleus. Ninety fields with immune infiltration were stained with the F4/80 antibody to determine which infiltrates contained macrophages.

For the detection of CD4+ and CD8+ lymphocytes, the tissues were embedded in Tissue Tek medium and frozen at $-70^{\circ} \mathrm{C}$. The sections were fixed with cold acetone and washed three times with PBS, followed by incubation with $0.3 \% \mathrm{H}_{2} \mathrm{O}_{2}$; after three additional washes with PBS, the sections were incubated with 2\% BSA for $1 \mathrm{~h}$. Anti-CD4+ (BD Pharmigen, Bedford, MA, USA) and anti-CD8+ antibodies (AbD serotec, Raleigh, NC, USA), or isotype controls (AbD serotec), were added and allowed to incubate overnight at $4^{\circ} \mathrm{C}$. After three washes with PBS, biotin-conjugated goat anti-rat IgG (Santa Cruz Biotechnology, Santa Cruz, CA, USA) was added for $90 \mathrm{~min}$ at room temperature. Finally, after three washes with PBS, streptavidin-peroxidase was used, and the reaction was developed with 3, 3'-diaminobenzidine. Sections were counterstained with H-E. Areas with brown histochemical staining were analyzed using Image J, version 1.42q (Wayne Rasband, NIH, USA) (http://rsb.info.nih.gov).

\section{Tissue Parasitism and Inflammation score}

Tissue parasitism was measured by counting the amastigote nests present in 96 microscope fields of each analyzed organ. Inflammation was scored by assessing the level of inflammatory infiltration in 236 fields in the small intestine and colon. Inflammatory infiltrates were scored using the following system: normal (0); mild, few infiltrates in the muscularis externa (1); moderate, diffuse infiltrates in muscularis externa and submucosa (2); severe, abundant infiltrates in muscularis externa, mucosa and submucosa (3) (Vizcaino, A. Master Thesis. 2007. UNAM). This score was based on a similar study that investigated $T$. cruzi- induced inflammation in the heart and skeletal muscle [19]. The observations described above were made using a light microscope (Nikon, Japan, Mod 022893), and a Nikon Coolpix 4300 was used to capture digital images.

\section{Measurement of cytokines}

For cytokine experiments, 6 mice were infected with the Ninoa strain, 6 with the Qro strain, and 6 were injected with PBS to serve as controls. For each infection, two independent experiments were performed. The experimental time point used for analysis was 21 days pi.

\section{Extraction of total RNA and preparation of cDNA}

Infected mice and controls were sacrificed at 21 days pi by an overdose of sodium pentobarbital, and the intestines were immediately removed. The small intestine and colon were each extensively washed with $0.1 \%$ DEPC-treated water, and the small intestine was then separated into three portions (duodenum, jejunum and ileum). A piece of the colon and pieces of each portion of the small intestine were homogenized to allow for the extraction of total RNA. Homogenization was performed in $1 \mathrm{ml}$ of the Trizol LS reagent, according to the manufacturer's recommendations (Invitrogen, Carsbald, CA, USA). Each RNA sample was resuspended in $50 \mu \mathrm{L} 0.1 \%$ DEPC water, and the amount of RNA in each sample was quantified using a Gene Quant Pro spectrophotometer (Pharmacia, Buckinghamshire, NA, England). The quality of the RNA was assessed by agarose gel electrophoresis, followed by staining with ethidium bromide and visualization with a UV transilluminator. Subsequently, $1 \mu \mathrm{g}$ of RNA from each sample was used for cDNA synthesis using reverse transcriptase (Applied Biosystems, Carsbald, CA, USA) according to the supplier's specifications. Finally, the amount of cDNA was measured using the Gene Quant Pro spectrophotometer (Pharmacia). 


\section{Real time quantitative RT-PCR assay for cyto- kine gene expression}

Real time quantitative PCR was performed in a 96-well microtiter plate (Perkin Elmer Applied Biosystems) format on an ABI PRISM 7000 Sequence Detection System (Applied Biosystems) according to the manufacturers' instructions. SYBR Green PCR Core Kit (Applied Biosystems) reagents were used to generate fluorescence signals during each PCR cycle via the 5' to 3' endonuclease activity of AmpliTaq Gold 14, which was used to provide real time quantitative PCR information. The sequences of the specific primers used in the RT-PCR analysis were designed in our laboratory using the Primer Express Application as primer design software, and the sequences are shown in Table 1.

Standard curves of quantified PCR products, as well as negative control, were included in each PCR run. The amount of the target cDNAs were obtained by interpolation the $\mathrm{Ct}$ value from the standard curve. To normalize gene expression, mRNA expression of the reference gene HPRT (hypoxanthine-guaninephosphoribosyltransferase) was measured. For each sample, both the housekeeping and target genes were amplified in triplicate. The PCR reaction conditions were optimized for each amplification, and the following cycles were used: $2 \mathrm{~min}$ at $50^{\circ} \mathrm{C}$ and $10 \mathrm{~min}$ at $95^{\circ} \mathrm{C}$, followed by 40 cycles of $15 \mathrm{~s}$ at $95^{\circ} \mathrm{C}$ and $1 \mathrm{~min}$ at $60^{\circ} \mathrm{C}$.

Real-time PCR efficiencies were determinate by amplification of a standardized dilution series, and slopes were calculated by lineal regression. The corresponding efficiencies (E) were then calculated according to the equation: $E=10^{(-1 / \text { slope) }}$. The transcript ratio of each cytokine in the samples was normalized to the transcriptional level of the houses-keeping HPRT and the relative expression ratio was calculated using the equation: ratio $=(\text { Etarget })^{\mathrm{CP} \text { control-CPsample / }}$

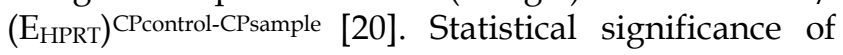
differences in the expression levels was evaluated using Two-tail unpared Student's t-test as previously reported. A p-value $<0.05$ was considered significant.

\section{Statistical Analysis}

The following results: parasitemia, the number of fields containing parasites, the presence of in- flammatory infiltrates and the number of fields containing macrophages were expressed as the media \pm standard deviation (SD). Significant differences were evaluated with a two-tailed Student's t-test (unpaired) for samples with different variances at a $95 \%$ confidence interval. The results for CD4+ and CD8+ cells are expressed as the media $\pm \mathrm{SD}$. The differences were analyzed using ANOVA with Tukey's post-test for multiple comparisons, and significant differences were considered to be values of $p \leq 0.05$. One way ANOVA was used to evaluate significant differences $(p<0.05)$ in cytokine gene expression for each section of the intestine used [21].

\section{RESULTS}

During the acute phase ( 15 and 21 days pi), mice infected with the Qro strain of T. cruzi presented with a hunched position, erect hair, tremors and hind limb paralysis. As the infection progressed to the chronic phase (90 days pi), the intensity of these symptoms decreased. These manifestations were not observed in mice infected with the Ninoa strain or in the uninfected control animals.

\section{Parasitemia}

The number of parasites present at each experimental time point for each strain of $T$. cruzi was counted in the blood and detected with immunohistological staining of the intestinal tract and heart of infected mice. At 15 days pi, there was a detectable parasitemia in the blood of mice infected with both strains. The peak of parasitemia in the serum was reached at 21 day pi for both strains (Figure 1A). After day 21, the parasitemia decreased in mice infected with both strains (data not shown). Days 15 and 21 pi are considered to be the acute phase in this mouse model. Significantly higher parasitemia was observed in mice infected with the Qro strain than in the mice infected with the non-virulent Ninoa strain. Nests of parasites were observed in the hearts of infected mice at days 15 and $21 \mathrm{pi}$, with a significantly higher number in the hearts of mice infected with the Qro strain (Figure 1B). In the chronic phase (90 days pi), no parasites were observed in mice infected with either strain.

TABLE I. Sequence of Primers

\begin{tabular}{llll}
\hline Cytokine family & Cytokine & 5'Primer & 3'Primer \\
\hline Th1 & IFN- $\gamma$ & 5'AAT GAA CGC TAC ACA CTG CAT 3' & 5' TGG CAG TAA CAG CCA GAA ACA 3' \\
Th1 & TNF- $\alpha$ & 5' GGG CAG GTC TAC TTT AGA GTC ATT G 3' & 5'GGC TGG GTA GAG AAT GGA TGA A 3' \\
Th2 & IL-4 & 5'GCA GAG ACT CTT TCG GGC TTT 3' & 5'TCA TTC ATG GTG CAG CTT ATC G 3' \\
reference Gene & HPRT & 5' GAA AGA CTT GCT CGA GAT GTC A 3' & 5' AGC ACA CAG AGG GCC ACA A 3' \\
\hline
\end{tabular}


Due to the fact that parasites were detected at 15 and 21 days pi, but not at 90 days pi, the level of parasitemia in the intestine was only measured at 21 days pi. Measurement of intestinal parasitaemia was carried out by histological and histochemical techniques. In the 3 segments of the small intestine, parasites were observed using a specific anti-T. cruzi antibody for immunohistochemical staining (Figure 1C). With this method, it was possible to observe small nests of parasites, distributed mainly in the muscular layer of the small intestine (Figure 2). In a minority of samples, large nests were observed in the duodenum (Figure 2 ). In contrast, large nests were observed in colon, and it was possible to observe them with $\mathrm{H}-\mathrm{E}$ staining (Figure 2). In mice infected with the Qro strain, the number of fields with parasites was higher in the colon than in the jejunum and ileum. This difference was not observed in the Ninoa-infected mice (Figure 1D). A difference was observed in the number of fields with parasites particularly in the colon when comparing the mice infected with the two different strains (Figure 1D).
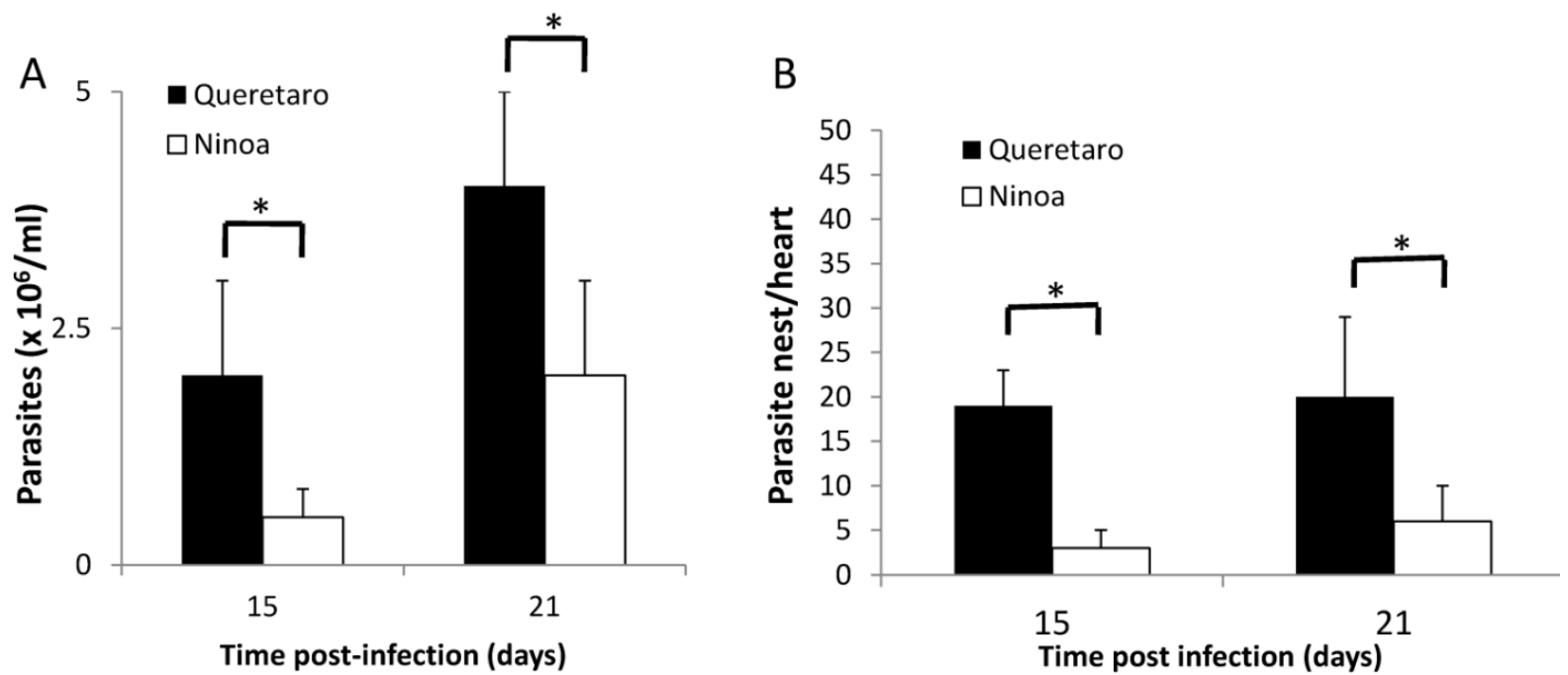

C

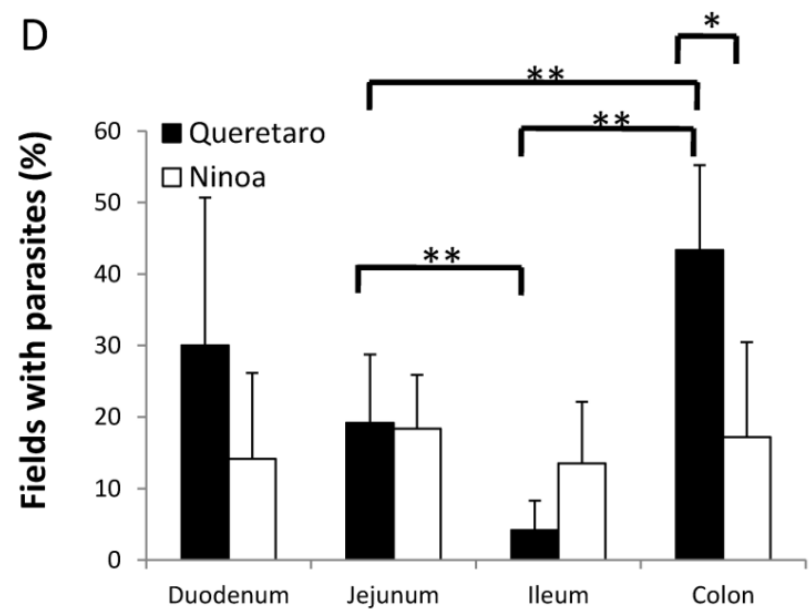

Figure I. Parasites in blood, heart and intestinal tract of mice infected with Tcl Méxican strains. A) Groups of three to six mice were infected with $T$. cruzi strains and parasite number in blood was evaluated by counting in a Neubauer chamber under an optical microscopy, t-student significant differences between strains $\left({ }^{*} \mathrm{p}<0.05\right)$ are shown. B) Heart sections, obtained as described in materials and methods, were $\mathrm{H}-\mathrm{E}$ stained and parasite nests were counted, significant differences between strains were found $(* p<0.05)$. C) Detection of parasites by immunofluorescence. Parasites were detected with a specific rabbit antibody against $T$. cruzi and then a FITC goat anti-rabbit IgG was added as described in materials and methods (green), nucleus and kinetoplast were stained with DAPI (blue). (Magnification x400). D) Percentage of fields with parasites in small intestine and colon ( 2 Idays pi) were determined as described in materials and methods, analysis by t-student two tails (unpaired) was done, significant differences were observed between strains $\left({ }^{*} \mathrm{p}<0.05\right)$ and between intestinal sections $(* * p<0.05)$. 
Duodenum
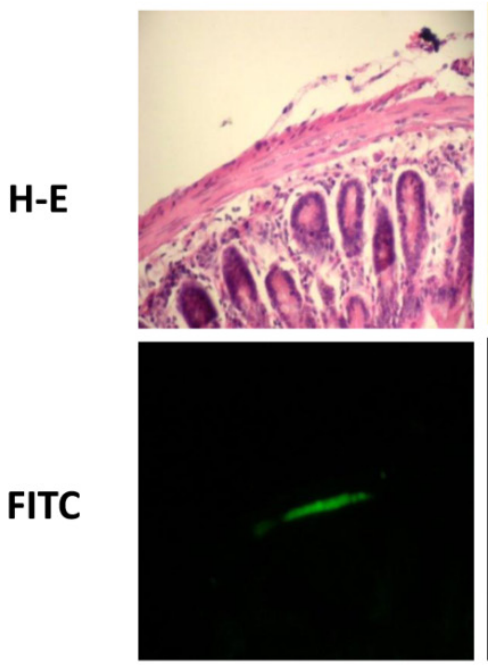

Jejunum
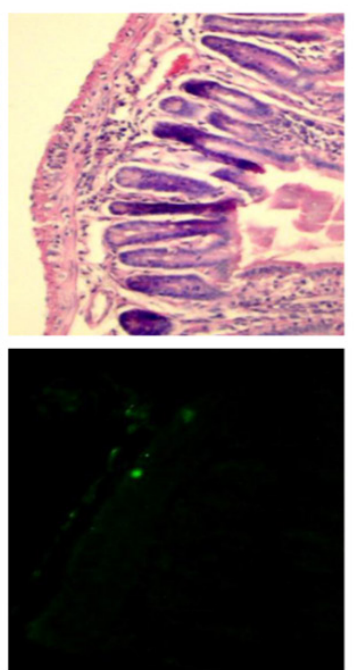

lleum
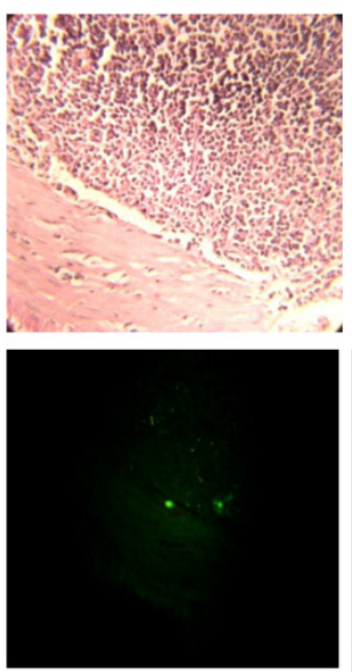

\section{Colon}
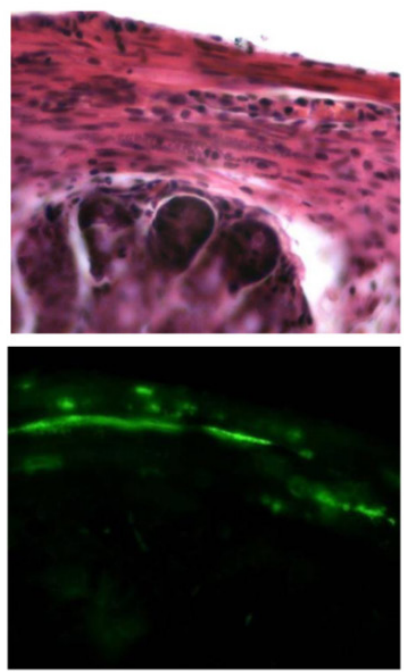

Figure 2. Parasite nests in different intestinal tract sections. Parasites nests in mice infected with $T$. cruzi Ninoa strain 21 days pi (duodenum, jejunum and ileum) or Queretaro strain 15 days pi (colon). Sections were stained by $\mathrm{H}$-E (upper panel) or parasite detected by immune-fluorescence using a rabbit serum against $T$. cruzi and a FITC goat anti-rabbit lgG as described in materials and methods (lower panel). Duodenum, jejunum and ileum (magnification $\times 200)$. Colon (magnification $\times 400)$.

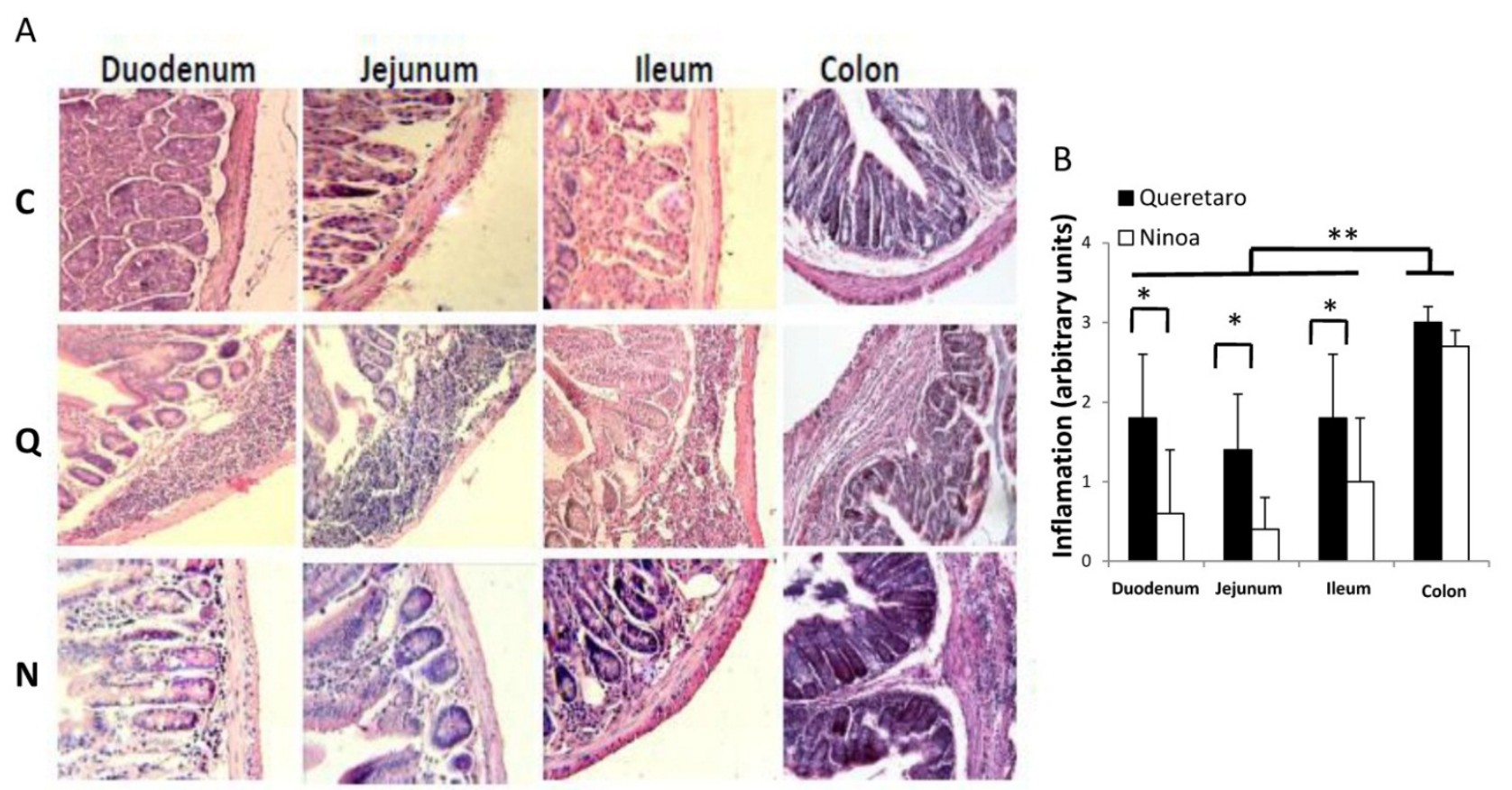

Figure 3. Inflammatory infiltrates in small intestine and colon from mice infected with $T$. cruzi 21 days pi. A) Sections of the intestinal tract stained with H-E. C) Mice uninfected, Q) Mice infected with Queretaro strain, N) Mice infected with Ninoa strain. (Magnification x200). B) Inflammation score in small intestine and colon was calculated as describe in material and methods and represented as media \pm SD. Statistical differences were determinate by t-student two tails (unpaired). Significant differences between strains $\left({ }^{*} \mathrm{p}<0.05\right)$ and between colon and small intestine $(* * p<0.05)$ were found.

\section{Inflammatory infiltrates}

In the small intestine of infected mice, diverse degrees of infiltration were observed. The level of inflammation ranged from small foci in the mucosa and submucosa layers (moderate inflammation) with the Ninoa strain to extensive inflammatory infiltrates from the submucosa to the muscularis externa (severe inflammation), which led to tissue damage and disruption of the intestinal structure in mice infected with the Qro strain (Figure 3A). In each of the three 
sections of the small intestine, significant differences were observed between the two strains (Figure 3B). In the colon, extended regions of inflammatory infiltration, hypertrophy of the muscular layer and fibrosis were observed in mice infected with both strains. The colon seems to be the intestinal compartment with the strongest inflammatory response, and significant differences were observed in both strains for the colon relative to the three sections of the small intestine (Figure 3B).

When CD4+ and CD8+ lymphocytes were evaluated by immunohistochemical staining in sections of the small intestine at 21 days pi, the most evident observation was the change in the distribution of these cells in the mice infected with the Qro strain. The uninfected control mice showed both types of cells, mainly in the intestinal villi. In contrast, in the mice infected with the Qro strain, extensive cell migration from this localization to the muscularis mucosa in the mucosal layer, to the circular and longitudinal muscular layers and to the muscularis externa was observed (Figures 4 and 5). This redistribution of CD4+ and CD8+ cells was not evident in mice infected with the Ninoa strain.

However, when the lymphocyte areas were quantified, no increase in CD4+ cells was observed. Only the areas with CD8+ cells were significantly more abundant in the jejunum of mice infected with the Qro strain. The other segments of the intestine did not show differences compared to the corresponding segments in control mice (Figure 6).

Because CD4+ and CD8+ cells did not seem to be the principal cells present in the inflammatory infiltrates in the small intestine, macrophages were also evaluated by immunohistochemical staining. In general, it was observed that the localization of macrophages varied between the sections (Figure 7A). Differences in the number of fields with macrophages were observed between sections of the small intestine, but not between the colon and the small intestine (Figure 7B). When the presence of macrophages was analyzed, a significant difference was only observed for the ileum between mice infected with the two different strains (Figure 7B).

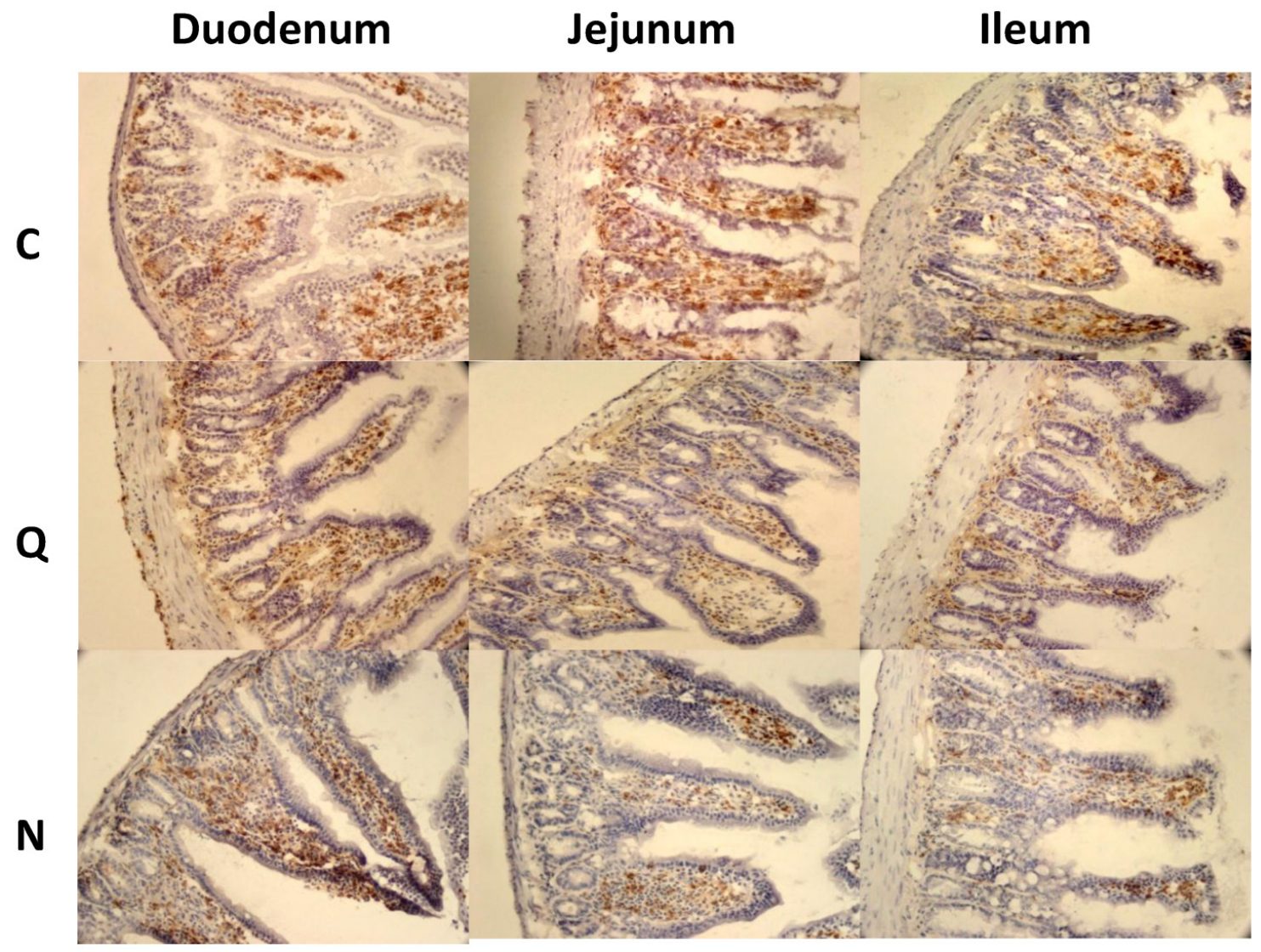

Figure 4. CD4+ Lymphocytes in small intestine from mice infected with $T$. cruzi 21 days pi. Sections from the small intestine: C) Mice uninfected, Q) Mice infected with Queretaro strain and N) Mice infected with Ninoa strain. CD4+ Lymphocytes were stained with anti CD4+ antibodies and revealed with 3,3-diaminobenzidine as described in materials and methods. Sections were counterstained with $H$-E. (Magnification $\times 200)$. 


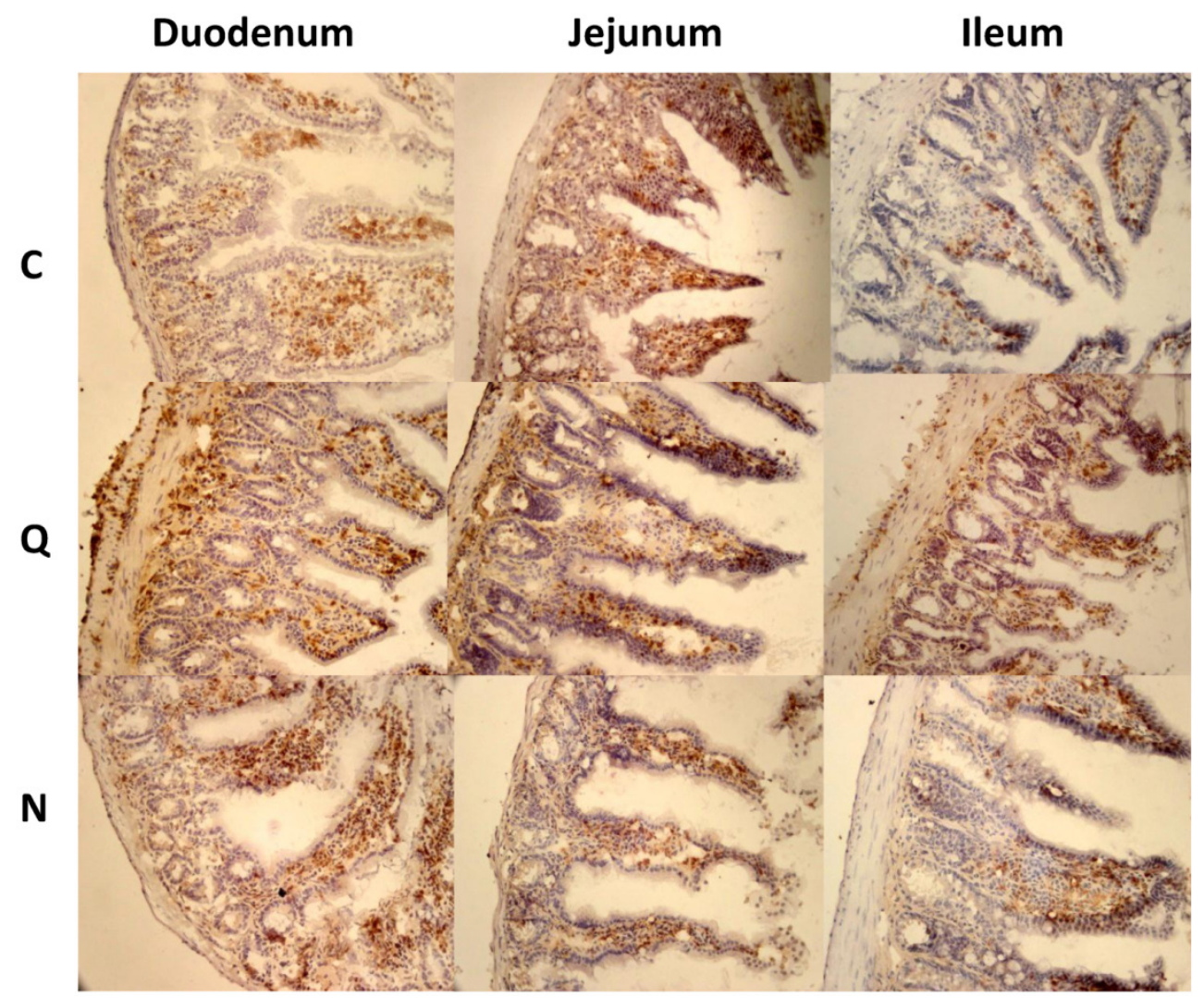

Figure 5. CD8+ Lymphocytes in small intestine from mice infected with T. cruzi 21 days pi. Sections from the small intestine from: C) Mice uninfected, Q) Mice infected with Queretaro strain and N) Mice infected with Ninoa strain. CD8+ Lymphocytes were stained with anti CD8+ antibodies and revealed with 3,3-diaminobenzidine as described in materials and methods. Sections were counterstained with $\mathrm{H}$-E. (Magnification $\times 200)$.

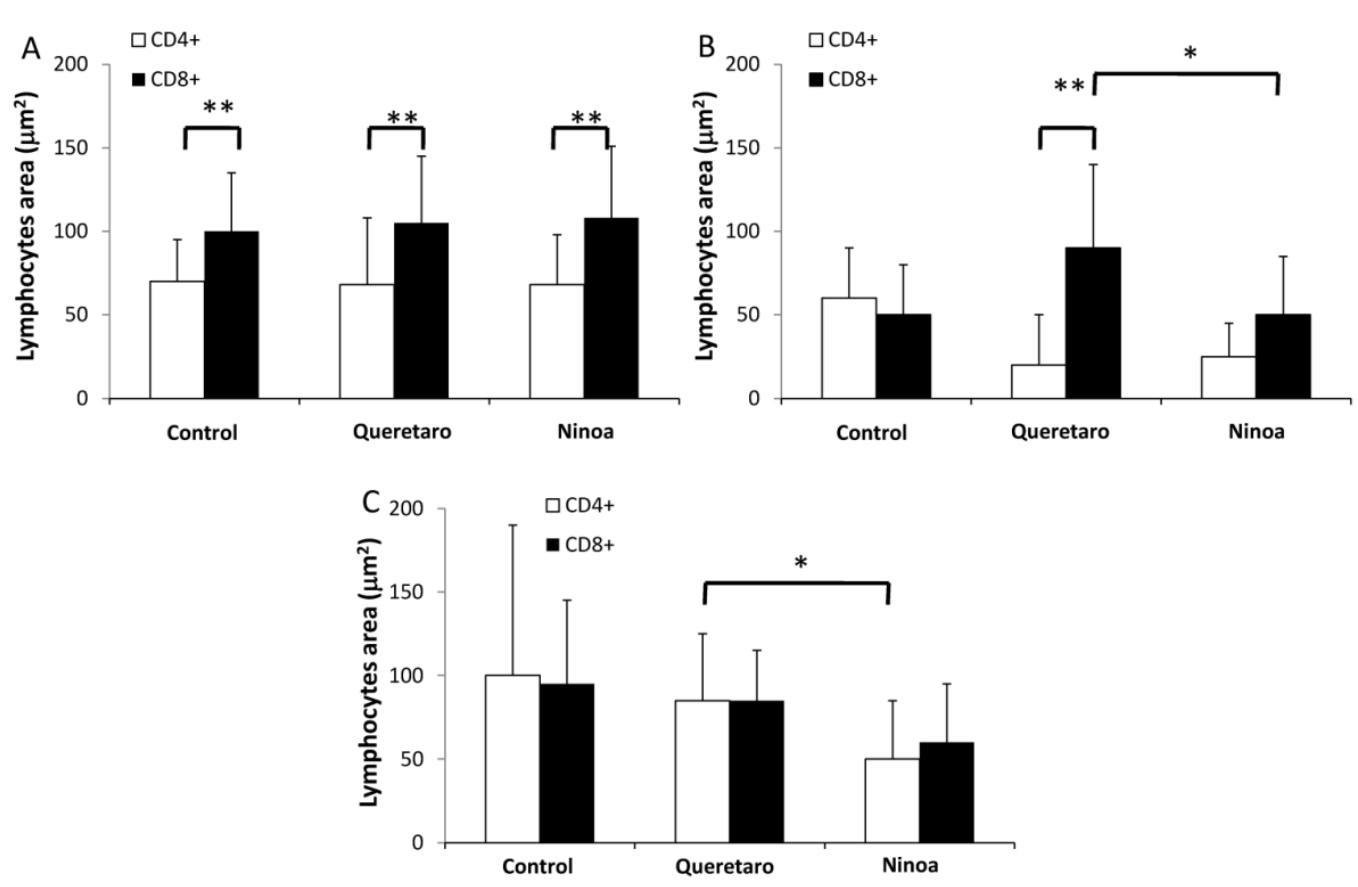

Figure 6. Quantification of areas with lymphocyte in mice uninfected and infected with $T$. cruzi strains $2 I$ days pi. Media value \pm SD were determined in: A) Duodenum, B) Jejunum and C) lleum of mice. Significant differences between strains $(* \mathrm{p}<0.05)$ or between cellular types $(* * \mathrm{p}<0.05)$ were found by one way ANOVA with Tukey post- test. 


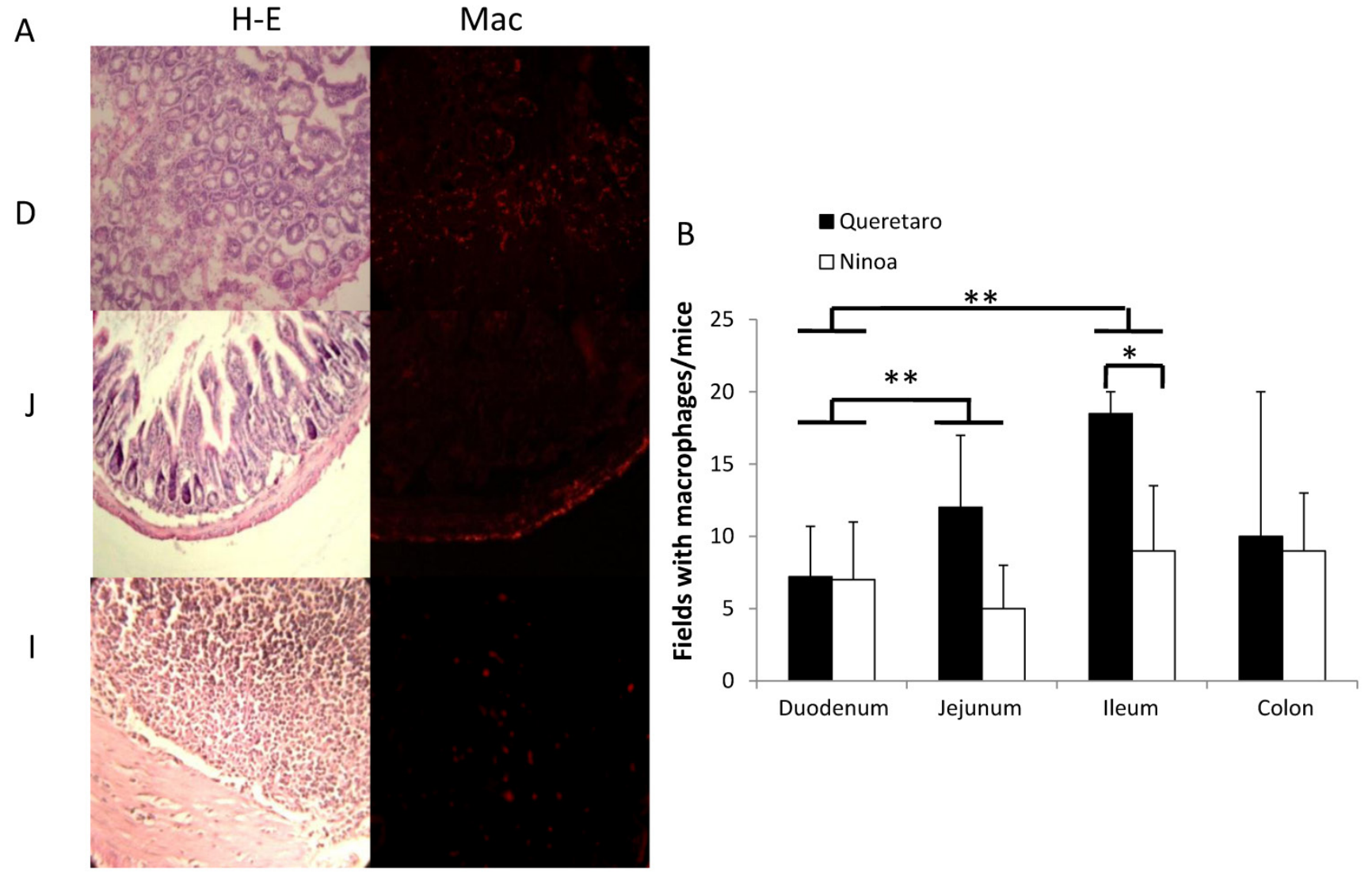

Figure 7. Macrophages in small intestine and colon from mice infected with T. cruzi. A) Sections of intestinal tract: D) Duodenum, J) Jejunum and I) lleon of mice infected with Ninoa strain $2 \mathrm{I}$ days pi were stained with H-E and macrophages (Mac) were detected with immunofluorescence as described in materials and methods. (Magnification $\times 200$ ). B) Quantification of fields with macrophage represented as media \pm SD of the number of fields with macrophages per mice at day 21 pi. Statistical differences were calculated by $t$-student two tails (unpaired). Significant difference between strains $(* p<0.05)$ and between sections $(* * p<0.05)$ were found.

The macrophages in the colon were abundant in certain areas, and their distribution was heterogeneous (Figure 8A, B, C). In some cases, the macrophages were found near to the parasites (Figure 8D), but in other cases the macrophages were not present in the immune infiltrate that surrounds the parasites (Figure $8 \mathrm{E})$. When the parasite nests that were infiltrated with immune cells were quantified, no differences were found between mice infected with the different strains. However, $57 \%$ of the infiltrates in mice infected with the Ninoa strain contained macrophages as part of the infiltrate. In contrast, only $27 \%$ of the infiltrated nests contained macrophages when mice infected with the Qro strain were evaluated (Figure $8 \mathrm{~F})$.

\section{Cytokine gene expression in the three seg- ments of the small intestine and in the colon at 2 I days pi.}

The gene expression of cytokines was increased relative to the controls in the small intestine and colon of mice infected with both strains. Differences in IFN- $\gamma$ and IL4 cytokines between strains were observed only in jejunum. Also, differences between sections of the intestinal tract occurred (Figure 9). IFN- $\gamma$ was significantly more expressed in jejunum of mice infected with the Qro strain than in duodenum, ileum and colon of mice infected with both strains (Figure 9A). In contrast, TNF-a gene expression was significantly more expressed in duodenum and colon than in jejunum (Figure 9B). The Th2 cytokine IL-4 was significantly higher in jejunum of mice infected with Qro strain than in the two other sections of the small intestine. Gene expression of this cytokine was also higher in colon of mice infected with Ninoa strain respect to ileum of mice infected with both strains (Figure 9C).

\section{DISCUSSION}

Chagas' disease is mainly an infection of cardiac and intestinal tissues. In Mexico, as in other countries of North and South America, the main pathology is 
the cardiac damage $[11,12]$. However, human studies indicate that intestinal Chagas' disease can also occur in Mexico [22, 23, 24]. A variety of symptoms have been observed in patients with Chagas' disease, depending on the type of immune response of the host, the genetic DTU of the T. cruzi strain, as well as the size of the inoculum. In Mexico, the main genetic DTU that infects humans is TCI $[25,26]$. Two Mexican TcI strains have been studied using the Balb/c murine model, and differences in the degree of infectivity and virulence, as well as the systemic immune response that they induced, were reported [18]. The tissue tropism of Mexican strains has not been extensively studied, but evidence of the presence of T. cruzi in organs other than the heart has been reported in murine models $[27,28]$. Furthermore, the possibility that the Mexican strains can reach the intestinal tract, establish an infection there, induce an inflammatory reaction and cause pathological damage has not been previously explored.
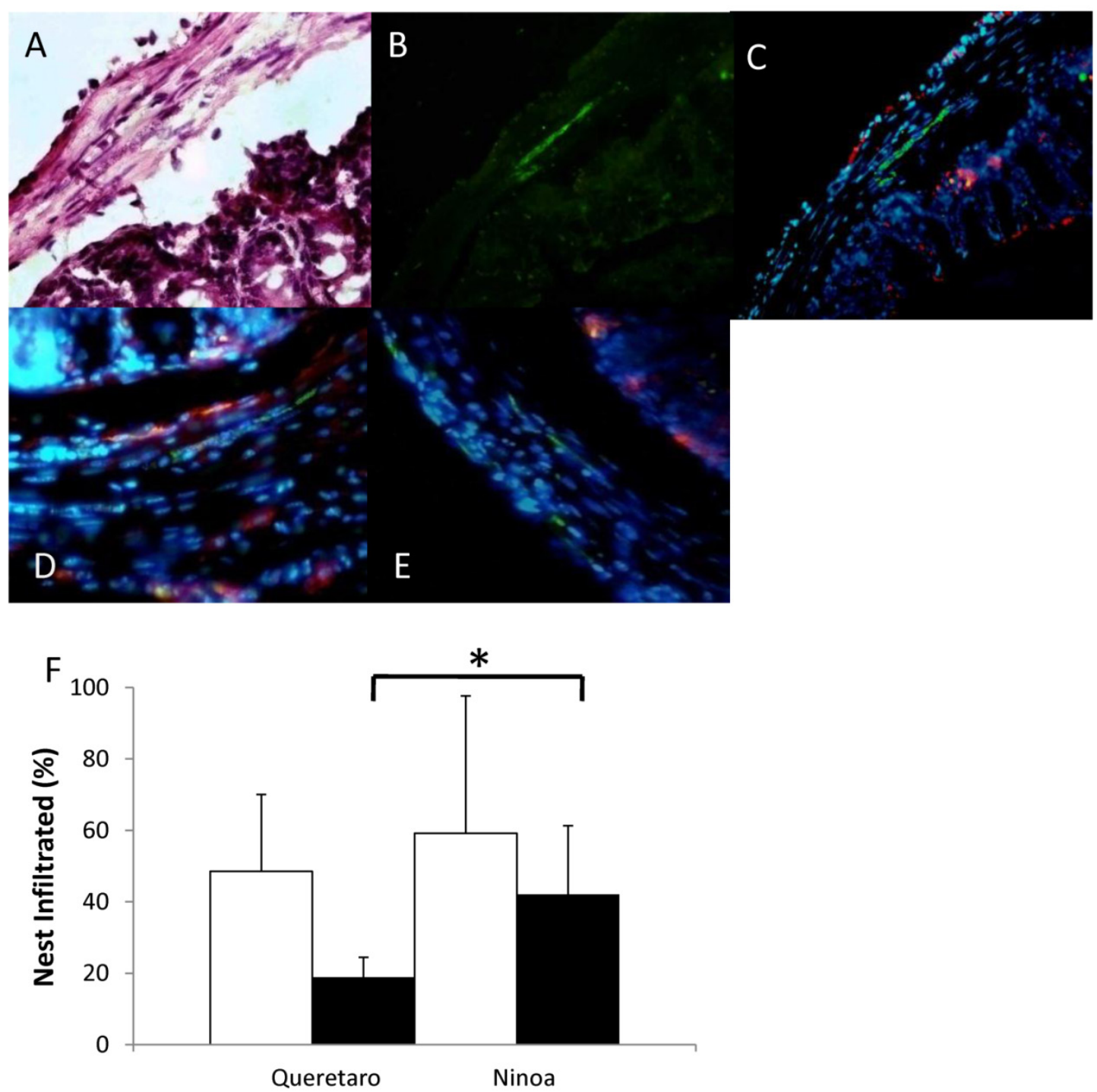

Figure 8. Macrophages and parasites in inflammatory infiltrates in colon. Areas with inflammatory infiltrates: A) Stained with $\mathrm{H}-\mathrm{E}$, B) Immunostained with a rabbit antibody against T. cruzi (green) and C) Merge of parasites (green) and macrophages (red) in colon of infected mice 15 days pi, nucleus were stained with DAPI (blue) (magnification x200), D) Nests of Ninoa strain with macrophages close to the parasites, E) Nests of Qro strain without the presence of macrophages near to the parasites (magnification $\times 400$ ). F) Quantification of immune infiltrates (white bars) and immune infiltrates with macrophages (black bars), $(* p<0.05)$. 

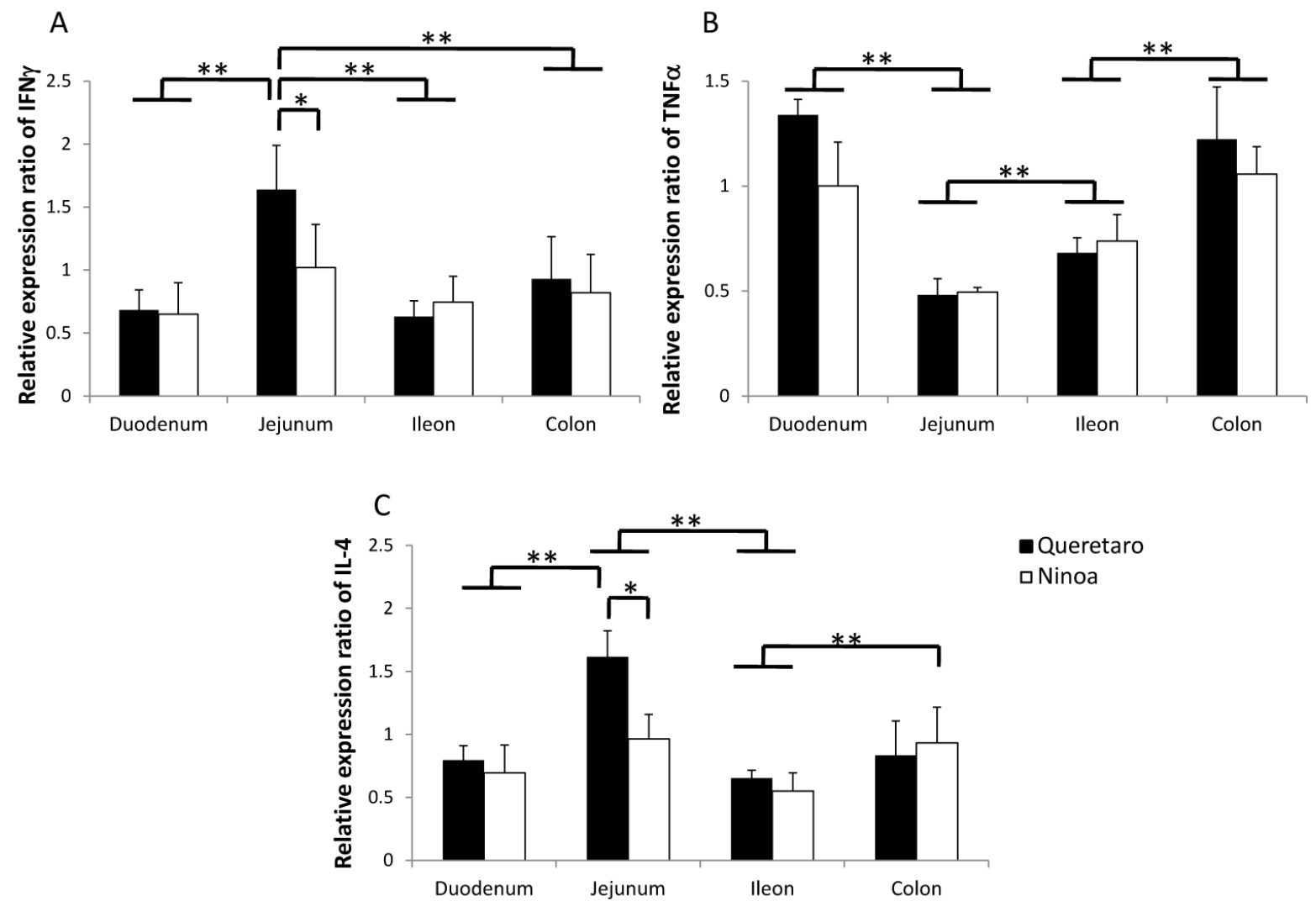

Figure 9. Gene expression of ThI and Th2 cytokines. Expression of INFy, TNFa and IL4 cytokines in sections of the small intestine and colon was analyzed by quantitative RT-PCR and the relative expression ratio was calculated as described in materials and methods. Six mice in two independent experiments were analyzed for each section. Significant differences between strains $(* p<0.05)$ and between sections $(* * p<0.05)$.

In this study, it was demonstrated that the Ninoa and Qro strains could invade the intestinal tract via the intaperitoneal route. The majority of the nests in the small intestine was small and could only be detected by immunofluorescence. The difficulty in detecting small parasite nests in the intestine may explain the absence of reports that show gastrointestinal infection by Mexican strains. The localization of parasites was irregular, and it varied depending on the intestinal segment analyzed. In general, parasites were located in the villi, submucosa and muscular layers, and also in the lymph nodes, but they were most abundant in the muscular layers. The number of fields with nests was the same in all three sections of the intestinal tract when mice were infected with the Ninoa strain. However, mice infected with the Qro strain showed significant differences in the number of fields with nests between the colon, ileum and jejunum. These results confirm the intestinal invasion capacity of the two strains and emphasize the different invasive properties of each strain. These results also corroborate the pathogenic nature of the Qro strain, which has been previously reported in studies of the heart muscle, although the two strains analyzed in this work belong to the same DTU TcI [18].

It is interesting to note that the colon was the more highly parasitized region of the intestinal tract in mice infected with the Qro strain. The reasons for this distribution of parasites are not clear, but it may be related to the dissimilar immune characteristics of the different segments of the intestine [29].

Immune infiltrates were more abundant in the colon than in the small intestine of mice infected with both strains. This difference is probably due to the fact that intraepithelial lymphocytes (IELs) have different characteristics in the small and large intestine. It has been demonstrated that the IELs of the large intestine are more similar to peripheral lymphocytes, and in addition to other characteristics, they are more proliferative than the IELs of the small intestine [16].

One interesting observation was the very low increase in CD4+ and CD8+ cells in the small intestine of infected mice. These $\mathrm{T}$ cells were thought to increase as part of a response to eliminate the parasite. In contrast, a clear redistribution of these cells, from 
the villi to the submucosa layer, was observed in mice infected with the Qro strain.

The presence of CD4+ and CD8+ cells corresponds with the regions where the Meissner and $\mathrm{Au}$ erbach plexuses are located. This CD4+ and CD8+ cell localization is not directly associated with the presence of the parasite; however, it can be associated with the damage to the neuronal plexuses mentioned above, and it may be the principal cause of pathological damage, as has been reported in patients with the neuropathologic injury observed in intestinal Chagas' disease [30] and mouse models [31]. This redistribution of cells was not observed in the small intestine of mice infected with the Ninoa strain.

Macrophages have been proposed to be the first line of defense against invading microorganisms [32]. In this study, macrophages were found to be distributed in the muscular layers, including the muscularis externa, and were dispersed along the entire submucosal layer, including the Peyer's patches. Interestingly, in mice infected with the Ninoa strain, the macrophages were located near the parasite and formed part of the inflammatory infiltrate that surrounds the amastigote nests. This was not seen in the nests of mice infected with the Qro strain, in which the majority of the immune infiltrates did not contain macrophages. This may provide an explanation for the higher number of parasites found in the colon of the Qro-infected mice. In the future, the presence of a secreted factor that avoids close contact between macrophages and Qro strain parasites will be investigated. The number of macrophages and $\mathrm{T}$ cells detected in the infiltrates was not sufficient to account for the entire composition of the inflammatory infiltrates. Therefore, the effects of these infections on other cells, such as NK cells, eosinophils and mast cells, should be studied because they have been reported in patients with megacolon [33].

It was also found that the expression of cytokine genes was higher at day 21 pi in mice infected with both strains, relative to the uninfected mice. The increase in immune mediators is related to the ability of the host to control infection because these mediators activate the cellular immune response in the infected tissue to kill the parasite, as has been observed in infected cardiomyocytes [34]. IFN- $\gamma$ and TNF- $\alpha$ were detected in the small intestine of mice infected with both strains. These cytokines can induce a Th1 response that benefits the host. Expression of IL-4 mRNA was observed in all three sections of the intestinal tract of mice infected with both strains. This cytokine can induce a Th2 response and can also function to regulate Th1 immune responses. In this study, the latter situation may be the case because the IL-4 expression controlled the Th1 response, which was correlated with the presence of the parasite in these tissues. Gene expression of IFN- $\gamma$, TNF- $\alpha$ and IL-4 was also found in the colon, but no differences were found in the colon between mice infected with the two different strains. This situation may explain the very similar appearances of the inflammatory infiltrates in this section.

It has been shown that in cardiac tissue infected with a Colombian strain of T. cruzi, there was an inflammatory infiltrate with a dominant presence of CD4+ $\mathrm{T}$ cells and an increase in the expression of IL-1 $\beta$, IL-12, TNF- $\alpha$, IFN- $\gamma$, MCP-1, MIP-1 $\alpha$, MIP-1 $\beta$ and MIP-2 during the acute phase of the infection [35, 36]. It has been reported that in macrophages treated with IFN- $\gamma$, there is an increase in IP-10 and MIG. Also, TNF- $\alpha$ induced an increase in the expression of IP-10, MIP-1 $\alpha$ and MCP-1, and these chemokines are involved in the production of nitric oxide (NO), which is the factor that kills the parasite inside macrophages [37]. However, it has been proven that NO can also damage neural cells, such as the cells that control intestinal motility, and this may cause the pathological condition called Chagasic enteropathy. $\mathrm{NO}$ is also involved in the peripheral denervation observed in the acute phase of experimental T. cruzi infection [38].

Another relevant issue is that epithelial cells of the small intestine can secrete inflammatory cytokines thus these cells could be another source of variability. In infections with other parasites, such as Toxoplasma gondii and Cryptosporidium paroum, this is the case, and it has been observed that there is an increase in the amounts of these cytokines and that they participate in the control of the parasitic infection $[39,40]$. Thus, the study of epithelial cells and their role in the inflammatory reaction observed in this mouse model should be studied in the future.

This work demonstrated that Mexican TcI strains can invade, establish and produce a robust immune response in the intestinal tract of Balb/c mice, as represented by extended immune infiltrates in the areas where the Meissner and Auerbach plexuses are located, and that macrophages may have an important regulatory presence mediated by cytokines. Further studies using this model will focus on other cell types, such as eosinophils, mast cells and NK cells, as well as immune mediators, including IL-17 and chemokines.

\section{Acknowledgments}

We thank Edgar Oaxaca-Hernández for his help in the design of primers for cytokines. Grant IN 229209 from DGAPA, UNAM and grant PICSA 10-130 from ICyTDF from the government of Mexico City partially supported this work. 


\section{Conflict of Interests}

The authors have declared that no conflict of interest exists.

\section{References}

1. WHO. Control of Chagas Disease; Technical Report Series 905. Geneva: WHO. 2002.

2. Gascon J, Bern C, Pinazo MJ. Chagas Disease in Spain, the United State and other non-endemic countries. Acta Trop. 2011; 115: 22-7.

3. Rodríguez-Coura J, Allbajar-Viñas P. Chagas disease: a new worldwide challenge. Nature. 2010. 465 (7301): S4-S5.

4. Zingales B, Andrade SG, Briones MRS, et al. A new consensus for Trypanosoma cruzi intraspecific nomenclature: second revision meeting recommends TcI to TcVI. Mem Inst Oswaldo Cruz. 2009; 104: 1051-4.

5. Bosseno MF, Barnabe C, Magallon-Castellum E, et al. Predominance of Trypanosoma cruzi Lineage I in Mexico. J Clin Microbiol. 2002; $40: 627-32$.

6. Añez N, Crisante G, da Silva FM, et al. Predominance of lineage I among Trypanosoma cruzi isolates from Venezuelan patients with different clinical profiles of acute Chagas' disease. Trop Med Int Health. 2004; 9: 1319-26.

7. Mejia-Jaramillo AM, Pena VH, Triana-Chavez O. Trypanosoma cruzi. Biological characterization of lineages I and II supports the predominance of lineage I in Colombia. Exp Parasitol. 2009; 121: 83-91.

8. Llewellyn MS, Miles MA, Carrasco HJ, et al. Genome-Scale multilocus microsatellite typing of Trypanosoma cruzi discrete typing units I reveals phylogeographic structure and specific genotypes linked to human infection. PLoS pathogens. 2009; 5: e1000410.

9. López-Olmos V, Pérez-Nasser N, Piñero D, et al. Biological characterization and genetic diversity of Mexican isolates of Trypanosoma cruzi. Acta Trop. 1998; 69: 239-254.

10. Espinoza B, Vera-Cruz JM, González H, et al. Genotypic and virulence correlation within Mexican stocks of Trypanosoma cruzi isolated from patients. Acta Trop. 1998; 70: 63-72.

11. Gloss G, Barrera M, Monteón VM, et al. Tripanosomiasis Americana y cardiopatia chagasica crónica en el Instituto Nacional de Cardiología Ignacio Chavez. Arch Inst Cardiol Méx. 1990; 60: 261-6.

12. Rassi A Jr, Rassi A, Marin-Neto J A. Chagas disease. Lancet. 2010; 375:1388-402.

13. Meneghelli UG. Chagasic enteropathy. Rev Soc Brasileña Med Trop. 2004; 37: 252-60.

14. Dutra WO, Gollob KJ. Current concepts in immunoregulation and pathology of human Chagas disease. Curr Opin Infec Dis. 2008; 21: 287-92.

15. Aprile LR, Troncon LE, Meneghelli UG et al. Small bowel bacterial overgrowth symdrome in chagasic megajejunum: report of 2 cases. Arq Gastroenterol. 1995; 32: 71-8.

16. Camerini V, Panwala C, Kronenberg M. Regional specialization of the mucosal immune system. J Immunol. 1993; 151: 1765-76.

17. Cronkhite RI, Michael J. Sub-compartmentalization of the gastrointestinal (GI) immune system determined with microbeads that differ in release properties. Vaccine. 2004; 22: 2106-15.

18. Espinoza B, Rico T, Sosa S, et al. Mexican Trypanosoma cruzi TCI strains with different virulence induce diverse humoral and cellular immune response in murine experimental infection. J Biomed Biotechnol. 2010;2010:890672.

19. Sun J, Tarleton RL. Predominance of CD8+ T lymphocytes in the inflammatory lesions of mice with acute Trypanosoma cruzi infection. Am Trop Med Hyg. 1993; 48: 161-9.
20. Pfaffl M. A new mathematical model for relative quantification in real-time RT-PCR. Nuc Ac Res. 2001; 29: 2002-7.

21. Rosner B. Fundamentals of Biostatistics. Pacific Grove CA, USA: Duxbury; 2000.

22. Salazar-Schettino PM, Tay J, Bucio MI, et al. First case of megaeshophagus with positive Trypanosoma cruzi serology. Salud Pública México. 1984; 26: 452-5.

23. Ocampo S, López J, Gálvez B, et al. Megaesófago chagásico con serología y xenodiagnóstico positivos. Rev Mexicana Gastroenterol. 1993; 58: 36-8.

24. Lozano-Kasten F, Hernández-Gutiérrez R, Kasten-Monge N, et al. Manifestaciones digestivas en la fase crónica de la enfermedad de Chagas. Cir Ciruj.1997; 65: 10-4.

25. Rangel-Flores H, Sánchez B, Mendoza-Duarte J, et al. Serological and parasitological demonstration of Trypanosoma cruzi infections in an urban central area of Mexico: Correlation with electrocardiographic alterations. Am J Trop Med Hyg. 2001; 65:887-95.

26. Ruíz-Sánchez R, De Leon M, Matta V, et al. Trypanosoma cruzi isolates from Mexican and Guatemalan acute and chronic chagasic cardiopathy patients belong to Trypanosoma cruzi I. Mem Inst Oswaldo Cruz. 2005; 100: 281-3.

27. Vera-Cruz J.M, Magallón-Gastelum E, Grijalva G, et al. Molecular diagnosis of Chagas disease and use of an animal model to study parasite tropism. Parasitol Res. 2003; 89: 480-6.

28. Melnikov VG, Fierro-Velasco F, Espinoza-Gómez F, et al. Pathological changes in lungs caused by Mexican isolates of Trypanosoma cruzi in the acute phase of infection in mice. Am J Trop Med Hyg. 2005; 73: 301-6.

29. Rezendiz-Albor A, Esquivel R, López-Revilla R, at al. Striking phenotypic and functional differences in lamina propia lymphocytes from the large and small intestine of mice. Life Sci. 2005; 76: 2783-803.

30. De Silveira ABM, Lemos EM, Adad SJ, et al. Megacolon in Chagas disease: a study of inflamatory cells, enteric nerves and glial cells. Hum Pathol. 2007; 38: 1256-64.

31. Arantes, R.M., Marche, H., Bahia, M.T., et al. Interferon- $\gamma$-induced Nitric Oxide causes intrinsic intestinal denervation in Trypanosoma cruzi-infected mice. Am J Pathol. 2004; 164: $1361-8$

32. Mosser, DM, Edwards JP. Exploring the full spectrum of macrophages activation. Nat Rev Imm.2008; 8: 958-69.

33. Da Silveira ABM, Adad SJ, Correa-Oñoveira R, et al. Morphometric study of eosinophils, mast cells, macrophages and fibrosis in the colon of chronic chagasic patients with and without megacolon. Parasitology. 2007; 134: 789-96.

34. Machado FS, Martins G.A, Aliberti JCS, et al. Trypanosoma cruzi infected cardiomyocytes produce chemokines and cytokines that trigger potent nitric oxide-dependent trypanocidal activity. Circulation. 2000; 102: 3002-8.

35. Talvani A, Ribeiro CS, Aliberti JCS, et al. Kinetics of cytokine gene expression in experimental chagasic cardiomyopathy: tissue parasitism and endogenous IFN- $\gamma$ as important determinants of chemokine mRNA expression during infection with Trypanosoma cruzi. Microbes Infect. 2000; 2: 851-66.

36. Santos-Lima EC, Garcia I, Vicentelli M H, et al. Evidence for a protective role of tumor necrosis factor in the acute phase of Trypanosoma cruzi infection in mice. Infect. Immun. 1997; 65: 457-65.

37. Villalta F, Zhang $\mathrm{Y}$, Bibb KE, et al. The cysteine-cysteine family of chemokines RANTES, MIP- $1 \alpha$, and MIP- $1 \beta$ induce trypanocidal activity in human macrophages via nitric oxide. Infection and immunity. 1998; 66: 4690-9.

38. Garcia SB, Paula JS, Giovanetti GS, et al. Nitric oxide is involved in the lesions of the peripheral autonomic neurons observed in the acute phase of the experimental Trypanosoma cruzi infection. Exp Parasitol; 1999; 93: 191-7. 
39. Ehigiator RH, Romagoli $\mathrm{P}$, Bogot $\mathrm{K}$, et al. Mucosal cytokine and antigen specific response to Cryptosporidium parvum in IL-12P40 knockout mice. Parasite Immul. 2005; 27: 17-28.

40. Kasper L, Courret N, Darche S. Toxoplasma gondii and mucosal immunity. Inter J Parasitol. 2004; 34: 401-9. 